\title{
Radiomics in oncology - uncovering tumor phenotype from medical images: a short introduction
}

\author{
Matea Pavic ${ }^{1}$, Janita E. van Timmeren ${ }^{1}$ \\ 1 Department of Radiation Oncology, University Hospital Zurich and University Zurich, Rämistrasse 100, 8091 Zurich, Switzerland
}

\begin{abstract}
Radiomics is a promising method to quantify and describe the tumor phenotype on medical images. High numbers of image features are extracted from medical images and can be used within a clinical decision support system by integrating this data with clinical and pathological variables. Herein, we give a short introduction into this image analysis method and present an overview on the workflow.
\end{abstract}

Keywords: Radiomics, Image analysis, Machine learning, Prognostic modelling, Clinical decision support system

Varying outcomes of anticancer treatment for the same tumor histology and site have led to new insights in cancer biology and oncological research. Heterogeneity between individual patients, their biology and tumors itself gave rise to the concept of precision oncology - aiming to tailor the treatment and give "the right patient or the right tumor the right treatment". However, heterogeneity is not only present between different tumors but also within a tumor and is recognized as a possible factor for resistance to anticancer treatment [1]. Medical images nowadays play a crucial role in oncology - not only for diagnostic but also for therapeutic purposes and are often acquired at multiple consecutive timepoints during a patient's course of disease. Besides the anatomy of the tumor and of normal tissue, additional information and data are captured in the images. A strong relationship between the macroscopic phenotype of a tumor, seen on these medical images, and the underlying cancer biology has been shown $[2,3]$. However, the human eye is not capable of capturing all the subtle differences and shades. This information can be extracted in form of features, referring to as "Radiomics". Taking it all together, radiomics is a promising method to display the spatial and temporal heterogeneity within tumors. Furthermore, in contrast to radiological reports, which nowadays are still descriptive and written in unstructured text form without many measurements, radiomics is able to perform a quantitative image analysis aiming at a comprehensive and reproducible characterization of medical images. By integrating these data with clinical variables, radiomics models have the potential to be used in a clinical decision support system (CDSS) to guide clinical decision-making. This mini review aims to give a very brief and short introduction to the concept of radiomics. Notably, even though this review focusses on its application in oncology, radiomics is also used in non-malignant disorders [4]. More detailed information on the individual workflow steps and a comprehensive overview has already been published [5, 6].

\section{Radiomics workflow}

Radiomics analysis, more precisely the working steps of preprocessing of images and feature extraction, is done using specific software. There are some open-source or commercially available software programs. However, many institutions use in-house developed software. Apparently, to develop such software on its own, coding skills are required. It is useful if the software can handle all types of imaging data such as CT, MRI or PET and is not limited in its scope. The software has to fulfill some standard criteria and is ideally benchmarked according to the Image Biomarker Standardization Initiative (IBSI) [7]. Fig. 1 gives an overview of a radiomics workflow.

Imaging (Fig. 1a): A great advantage of radiomics is that medical images, the mainstay of a radiomics analysis, are regular part of the clinical routine. Yet, the use of routinely acquired images poses also a challenge as variability in scanner, scanning methods or image

This article is licensed under the Creative Commons Attribution 4.0 International License. To view a copy of this license, visit http://creativecommons.org/licenses/by/4.0/ or send a letter to Creative Commons, PO Box 1866, Mountain View, CA 94042, USA. Creative Commons Attribution 4.0 International license. $($ (c) EY 
a)

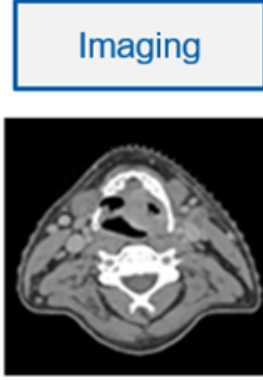

CT, PET, MRI, ... c)

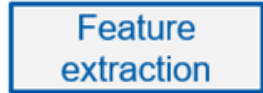

b)

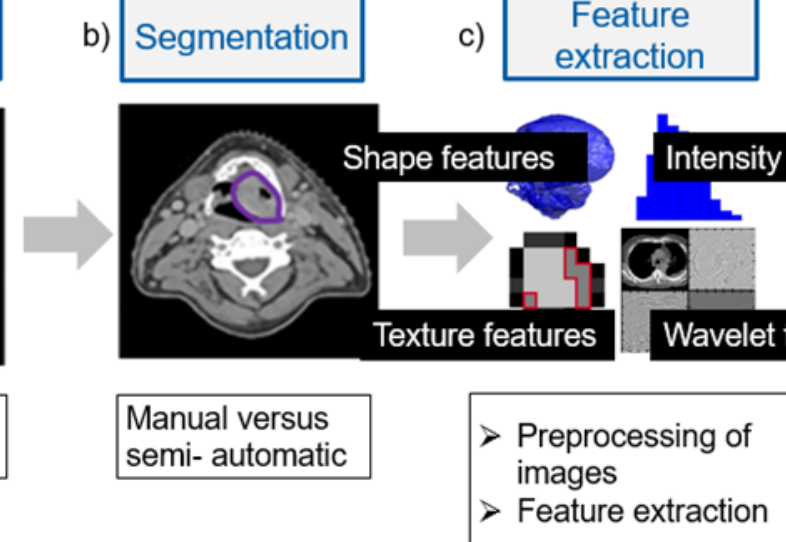

d) Data analysis

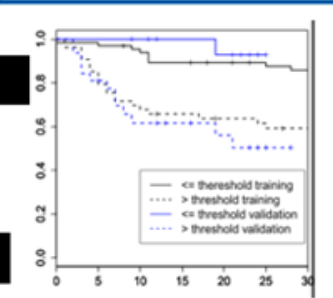

$>$ Feature selection $>$ Model training

$>$ Integration with other variables

Performance evaluation Model validation

Figure 1. Traditional clinical procedures.

reconstruction methods influence the results of a radiomics analysis [8]. However, imaging will remain nonstandardized, asking for correction and harmonization procedures that use for example information acquired from phantom studies [9].

Segmentation (Fig. 1b): Segmentation, i.e. definition of regions of interest (ROI) is a critical step in radiomics as features are extracted from these segmented areas. Intra- and interobserver variability in contouring ROIs is a well-known issue and influences results of radiomics analysis - therefore, ideally the ROI is segmented by different observers in order to extract only stable features irrespective of differences in segmentation [10]. Semi-automatic and automatic contouring methods were developed in the last years to escape this issue and to reduce the immense workload related to segmentation of structures [11]. However, (semi-)automatic contouring is prone to errors, especially in regions with artifacts or much noise and in case of an uncommon appearance of the ROI (e.g. anomaly).

Preprocessing of images (Fig. 1c): The routinely acquired images first have to undergo a preprocessing workflow. One step is the resampling of images to equally sized voxels using different interpolation methods such as linear or cubic interpolation. The images can either be up-sampled to smaller voxel sizes or down-sampled to larger voxel sizes. Image discretization refers to grouping the intensity-levels of an image into a predefined interval (i.e. bin), where one can choose to either fix the bin size or the number of bins and is performed in order to reduce noise while simultaneously preserving relevant features. To limit the analysis to the tissue interested in, e.g. to remove air and bone, a range of Hounsfield units can be applied.
Feature extraction (Fig. 1c): A clear definition, nomenclature and standardization of the features is a prerequisite for the reproducibility of a quantified image analysis by radiomics and is given by the IBSI $[7,12]$. The extracted features are typically divided in four subgroups: shape, intensity, texture and wavelet features. Shape features quantify the geometry of the segmented lesion. Intensity features look at the image intensities -represented by a histogram. Texture features describe how these intensities change from voxel to voxel. Finally, wavelet features are derived from filtered reconstructions of the images. By applying various filters, numerous wavelet features can be generated.

Feature selection (Fig. 1d): As stated above, a large number of features can be calculated from one single ROI, asking for a method, which allows to identify the relevant features for the clinical question. Use of all calculated features would lead to overfitting, which means that the generated radiomics model represents too closely the data set and fails on new, so far unseen, data. One predictor per ten events is considered to be the maximum for logistic and Cox models $[13,14]$. Consequently, there is a need to remove redundant features and reduce dimensionality, aiming to capture the essence of the data in a lower dimensional space. This is done by removing features with a lot of missing values or low variance and remove highly correlated features, i.e. feature selection. Additionally, a transformation algorithm to further reduce dimensionality is often applied, for instance principal component analysis [15].

Outcome modelling and integration with other variables (Fig. 1d): After selection of relevant features, a mathematical model is built with the aim to generate a 
predictive or prognostic model for a defined "clinical event" by combining the radiomics features with clinical, pathological or other parameters - thereby being able to support clinical decision-making. Different statistical or machine learning algorithms can be used for this purpose: e.g. linear regression, Cox proportional hazards model, support vector machines etc. [16, 17].

Performance evaluation (Fig. 1d): The performance of the generated radiomics model can be assessed using different methods, e.g. the area under receiver operator characteristic curve (AUC), sensitivity, specificity or accuracy. The distinct models can be compared by conventional statistical tests such as student t-test, Wilcoxon signed-rank test or others, depending on the metrics used. If multiple testing was performed, one should correct for that by applying one of the correction methods [18]. Additionally, the agreement between observed outcome and predicted outcome should be assessed - referred as calibration. For more details please refer to comprehensive literature on that topic $[16,19]$.

Model validation (Fig. 1d): The generated model should be validated on unseen data, preferably on an independent data set. Whenever possible, the model should be validated on a data set from a different institution (external validation) as this would be the reality if the model was applied in clinical routine and therefore enhances the credibility of a prediction model [20].
However, more commonly, the data set stems from the same institution (internal validation).

\section{Outlook}

A prerequisite for successful implementation of radiomics as a prognostic or predictive model into clinical routine in the future is standardization of the methodology. Studies performed should follow some important principles as provided by a checklist for the transparent reporting of a multivariable prediction model for individual prognosis or diagnosis (TRIPOD) and the radiomics quality score (RQS) [6, 19]. However, as not all of the radiomics steps can be standardized, compensation strategies have to be implemented. Imaging for example will remain non-standardized, which means that correction and harmonization procedures are needed, using information acquired from phantom studies [9]. Another key step before release of a radiomics model as a validated decision support tool is validation of its performance on a prospective patient cohort. In conclusion, radiomics has the potential to play a key role in a clinical decision support tool in precision oncology in future - however, the road to realize this vision is still a long one.

\section{References}

1. Dagogo-Jack I, Shaw AT: Tumour heterogeneity and resistance to cancer therapies. Nat Rev Clin Oncol 2018, 15(2):81-94

2. Panth KM, Leijenaar RT, Carvalho S, Lieuwes NG, Yaromina A, Dubois L, Lambin P: Is there a causal relationship between genetic changes and radiomicsbased image features? An in vivo preclinical experiment with doxycycline inducible GADD34 tumor cells. Radiotherapy and oncology : journal of the European Society for Therapeutic Radiology and Oncology 2015, 116(3):462-466.

3. Yeh AC, Li H, Zhu Y, Zhang J, Khramtsova G, Drukker K, Edwards A, McGregor S, Yoshimatsu T, Zheng Y et al: Radiogenomics of breast cancer using dynamic contrast enhanced MRI and gene expression profiling. Cancer Imaging 2019, 19(1):48.

4. Neisius U, El-Rewaidy H, Nakamori S, Rodriguez J, Manning WJ, Nezafat R: Radiomic Analysis of Myocardial Native $\mathrm{T}(1)$ Imaging Discriminates Between Hypertensive Heart Disease and Hypertrophic Cardiomyopathy. JACC Cardiovasc Imaging 2019, 12(10):1946-1954.
5. van Timmeren JE, Cester D, TanadiniLang S, Alkadhi $\mathrm{H}$, Baessler B: Radiomics in medical imaging-"how-to" guide and critical reflection. Insights into imaging 2020, 11(1):91.

6. Lambin P, Leijenaar RTH, Deist TM, Peerlings J, de Jong EEC, van Timmeren J, Sanduleanu S, Larue R, Even AJG, Jochems A et al: Radiomics: the bridge between medical imaging and personalized medicine. Nat Rev Clin Oncol 2017, 14(12):749-762.

7. Zwanenburg A, Vallières $M$, Abdalah MA, Aerts HJWL, Andrearczyk V, Apte A, Ashrafinia S, Bakas S, Beukinga RJ, Boellaard R et al: The Image Biomarker Standardization Initiative: Standardized Quantitative Radiomics for HighThroughput Image-based Phenotyping. $0(0): 191145$.

8. Mackin D, Fave X, Zhang L, Fried D, Yang J, Taylor B, Rodriguez-Rivera E, Dodge C, Jones AK, Court L: Measuring Computed Tomography Scanner Variability of Radiomics Features. Invest Radiol 2015, 50(11):757-765.

9. Ger RB, Zhou S, Chi PM, Lee HJ, Layman RR, Jones AK, Goff DL, Fuller $\mathrm{CD}$, Howell $\mathrm{RM}, \mathrm{Li} \mathrm{H}$ et al: Comprehensive Investigation on
Controlling for CT Imaging Variabilities in Radiomics Studies. Sci Rep 2018, 8(1):13047.

10. Pavic M, Bogowicz M, Wurms X, Glatz $\mathrm{S}$, Finazzi T, Riesterer O, Roesch J, Rudofsky L, Friess M, Veit-Haibach P et al: Influence of inter-observer delineation variability on radiomics stability in different tumor sites. Acta oncologica (Stockholm, Sweden) 2018, 57(8):1070-1074.

11. Sharp G, Fritscher KD, Pekar V, Peroni $\mathrm{M}$, Shusharina N, Veeraraghavan $\mathrm{H}$, Yang J: Vision 20/20: Perspectives on automated image segmentation for radiotherapy. 2014, 41(5):050902.

12. Alex Zwanenburg SL, Martin Vallières, Steffen Löck: Image Biomarker Standartisation Initiative.

13. Concato J, Peduzzi P, Holford TR, Feinstein AR: Importance of events per independent variable in proportional hazards analysis. I. Background, goals, and general strategy. J Clin Epidemiol 1995, 48(12):1495-1501.

14. Peduzzi P, Concato J, Feinstein AR, Holford TR: Importance of events per independent variable in proportional hazards regression analysis. II. Accuracy and precision of regression estimates. $\mathbf{J}$ 
Clin Epidemiol 1995, 48(12):1503-

1510.

15. Jolliffe IT, Cadima J: Principal component analysis: a review and recent developments. 2016 374(2065):20150202.

16. Steyerberg EW, Vickers AJ, Cook NR, Gerds T, Gonen M, Obuchowski N, Pencina MJ, Kattan MW: Assessing the performance of prediction models: a framework for traditional and novel measures. Epidemiology 2010, 21(1):128-138.

17. Wei L, Osman S, Hatt M, El Naqa I: Machine learning for radiomics-based multimodality and multiparametric modeling. Q J Nucl Med Mol Imaging 2019, 63(4):323-338.

18. Noble WS: How does multiple testing correction work? Nat Biotechnol 2009, 27(12):1135-1137.

19. Moons KG, Altman DG, Reitsma JB, Ioannidis JP, Macaskill P, Steyerberg EW, Vickers AJ, Ransohoff DF, Collins GS: Transparent Reporting of a multivariable prediction model for Individual Prognosis or Diagnosis (TRIPOD): explanation and elaboration. Ann Intern Med 2015, 162(1):W1-73.

20. Debray TP, Vergouwe Y, Koffijberg H, Nieboer D, Steyerberg EW, Moons KG: A new framework to enhance the interpretation of external validation studies of clinical prediction models. J Clin Epidemiol 2015, 68(3):279-289. 